\title{
Feasibility and safety of 4 weeks of blood flow-restricted exercise in an individual with tetraplegia and known autonomic dysreflexia: a case report
}

\author{
Søren Krogh $\mathbb{1}^{1,2} \cdot$ Anette B. Jønsson $^{1,2} \cdot$ Jørgen Vibjerg $^{1} \cdot$ Kaare Severinsen $^{1} \cdot$ Per Aagaard ${ }^{3} \cdot$ Helge Kasch $^{1,2}$
}

Received: 18 May 2020 / Revised: 25 August 2020 / Accepted: 25 August 2020

(c) International Spinal Cord Society 2020

\begin{abstract}
Introduction Blood flow-restricted exercise (BFRE) appears to hold considerable potential in spinal cord injury (SCI) rehabilitation, due to its ability to induce beneficial functional changes and morphological alterations from low-intensity, low-load exercise. However, it remains unclear if this training approach is feasible and safe in individuals with autonomic dysreflexia (AD).

Case presentation A 23-year-old male with traumatic, cervical (C6), motor-complete (AIS: B) SCI and diagnosed AD completed eight sessions of BFRE for the upper extremities over 4 weeks. Blood pressure and heart rate recordings and perceptual pain responses were collected repeatedly during exercise. Blood samples were drawn pre- and post-training. Training was carried out in a neurorehabilitation hospital setting with appertaining medical staff readiness, and was supervised by a physiotherapist with expertise in $\mathrm{AD}$ in general as well as prior knowledge of the present patient's triggers and symptoms. Four incidences of $\mathrm{AD}$ (defined as systolic blood pressure increase $>20 \mathrm{mmHg}$ ) were recorded across all training sessions, of which one was symptomatic. The patient's blood profile did not change considerably from pre- to posttraining sessions. Self-reported average pain during training corresponded from "mild" to "moderate".

Discussion The patient was able to perform 4 weeks of BFRE, but encountered episodes of AD. Similarly, two AD episodes were registered during a single conventional, free-flow resistance training session. Evidence from clinically controlled safety studies is needed in order to establish if and how BFRE can be applied in a rehabilitation strategy in SCI individuals with neurological level of injury at or above T6 level.
\end{abstract}

\section{Introduction}

Cervical spinal cord injury (SCI) is a catastrophic injury that leads to complications in numerous bodily systems, including locomotor functioning. Consequently, tetraplegia reduces independence and quality of life and leads to sedentarism, physical deconditioning and increased risk of secondary health conditions [1]. Recovery of upper

Søren Krogh

sokrje@rm.dk

1 Spinal Cord Injury Centre of Western Denmark, Regional Hospital Viborg, Viborg, Denmark

2 Department of Clinical Medicine, Aarhus University, Aarhus, Denmark

3 Department of Sports Science \& Clinical Biomechanics, University of Southern Denmark, Odense, Denmark extremity motor function is reported as the main priority for individuals with tetraplegia [2-4]; and while some function is regained during conventional rehabilitation, considerable upper extremity impairment often persists following discharge [5]. In addition, rehabilitation appears even less rewarding for individuals with motor-complete injuries [5]. Thus, a need exists for novel therapeutic approaches in this population.

Blood flow-restricted (BFR) resistance exercise (BFRE), in which low-load (20-50\% of 1-repetition-maximum) muscle contractions are performed under conditions of partial restriction of blood flow to the working muscle, has been shown to increase human skeletal muscle mass and strength to a degree that is comparable to that obtained by conventional heavy-load resistance training in healthy subjects [6]. Restriction of blood flow is typically effected by inflating a pneumatic cuff at the proximal end of the limb being trained. The potential ability to induce morphological and functional changes through BFR low-load muscle 
loading appears highly relevant in SCI rehabilitation, where atrophy, impaired neuromuscular endurance and various degrees of spasticity makes heavy-load resistance training impractical and often unattainable. Although previous case reports demonstrate functional improvements following BFRE in individuals with other neuromuscular diseases (myositis [7, 8] cerebral palsy [9], sarcopenia [10]), the technique is not well established as a rehabilitation approach.

Only a single previous study [11] has investigated the effect of BFRE in persons with SCI. Nine individuals with incomplete tetraplegia underwent 6 weeks of BFR combined with neuromuscular electrical stimulation (NMES) as training for the wrist extensors. Following the intervention period, cross-sectional area (CSA) of the extensor carpi radialis longus muscle increased by $15 \%$, to reach a $17 \%$ greater CSA than that of the contralateral control arm that underwent a similar NMES protocol without BFR [11]. No changes in CSA were seen in the extensor digitorum communis muscle. The authors also reported functional improvements in the NMES + BFR hand only, in terms of grasp and release test performance (time to complete grasping, moving and releasing a peg over a $60 \mathrm{~cm}$ distance; $1 \pm 0.4$ vs. $0.66 \pm 0.16 \mathrm{~s}, p=0.025$ pre vs. post).

Although excessive, unaccustomed, high-volume BFRE may lead to skeletal muscle damage to a degree indicative of rhabdomyolysis [12], the safety of progressively adjusted BFRE has been relatively well established in healthy, ablebodied individuals as well as in individuals with a variety of physical conditions such as cerebrovascular-, orthopaedic-, cardiac-, respiratory- and neuromuscular diseases, diabetes and hypertension [13, 14]. However, in individuals with $\mathrm{SCI}$ ischaemia and pain associated with BFRE may trigger autonomic dysreflexia (AD). AD is a potentially lifethreatening complication for individuals with neurological level of injury at or above the T6 level [15]. Therefore, the safety of this training approach needs to be further addressed in SCI. Stavres et al. [16] investigated hemodynamic changes, risk factors of deep-vein thrombosis (DVT) and the prevalence of $\mathrm{AD}$ during an acute bout of BFRE for the lower limbs in nine individuals with SCI (seven tetraplegics, two paraplegics). During a single exercise $(3 \times 10$ repetitions of unilateral leg extension) and after $15 \mathrm{~min}$ follow-up, the subjects showed no signs of AD or elevated risk factors for DVT formation. However, the relatively low cuff occlusion pressures used (range: 39-110, mean: $60.1 \mathrm{mmHg}$ ) and the high motor function status of their participants (all AIS: D) limit the dissemination of these results for the general SCI population. Thus, more knowledge is needed about how BFRE may affect SCI individuals with various degrees of $\mathrm{AD}$ and neurological deficits.

The present case report is the first to describe the safety and feasibility of a BFRE training intervention in an SCI individual with a concurrent history of $\mathrm{AD}$, using voluntary muscle activation.

\section{Case presentation}

The participant was a 23-year-old male patient admitted for neurorehabilitation at an SCI rehabilitation hospital. The anthropometrics and injury characteristics of the participant are presented in Table 1. At study initiation, the participant had been admitted for 9 months. No motor function was preserved below $\mathrm{C} 7$, whereas moderate function remained bilaterally in the upper extremities (Manual Muscle Test (MMT) scores are presented in Table 1). One month prior to initiation of the training programme the participant had a 24-h ambulatory blood pressure monitoring [17] (24-hABPM) performed. The participant presented with $\mathrm{AD}$ manifested as (1) bladder-distension-induced hypertension and tachycardia and (2) exercise-induced hypertension with compensatory bradycardia. In addition, he presented with autonomic dysfunction manifested as chronic hypotension and orthostatic hypotension. The participant completed 4 weeks of twice-weekly BFRE of the upper extremities for a total of eight sessions. The cuff (Cylindrical Tourniquet Cuff 90 mm, Zimmer Surgical, Inc., Dover, OH, USA) was systematically positioned at the most proximal end of the upper arm so that the most proximal edge of the cuff made contact with the anterior axillary fold, and inflated to a pressure of $100 \mathrm{mmHg}$ using a computerized inflation device (Portable Tourniquet System II, Delfi Medical, Vancouver, Canada). The specific level of cuff pressure was

Table 1 Anthropometrics and injury characteristics of the participant.

\begin{tabular}{ll}
\hline Age & 23 \\
Bodyweight (kg) & 55 \\
Height $(\mathrm{cm})$ & 178 \\
BMI & 17.4 \\
Neurological level of injury & $\mathrm{C} 6$ \\
AIS classification & $\mathrm{B}$ \\
Aetiology & Traumatic \\
Time since injury (months) & 11 \\
Zone of partial preservation & \\
R-motor & $\mathrm{C} 7$ \\
L-motor & $\mathrm{C} 7$ \\
R-sensory & $\mathrm{S} 5$ \\
L-sensory & $\mathrm{S} 5$ \\
Manual muscle test scores & $(\mathrm{R} / \mathrm{L})$ \\
Shoulder abductors & $5 / 5$ \\
Elbow flexors & $5 / 5$ \\
Wrist extensors & $3 / 3$ \\
Elbow extensors & $2+/ 2$ \\
\hline
\end{tabular}


selected based on previous training protocols documented to improve upper extremity function and morphology in able-bodied subjects [18]. Three sets to failure (15-25 repetitions per set) were performed for each exercise with $45 \mathrm{~s}$ of rest between sets. The upper extremities were trained unilaterally, and all exercises were completed for the left arm before training the right arm. Cuff release was allowed between exercises only (separated by 3 min rest). Exercises were (in order): horizontal triceps extensions, biceps curls, and wrist extensions. The participant performed all exercises seated incline in his electrical wheelchair. During biceps curls, a $3 \mathrm{~kg}$ sandbag was secured to the palm of the participant's hand; triceps and wrist extensions were performed without additional load. For safety reasons, all training sessions were carried out in the participant's room at a neurorehabilitation hospital with appertaining medical staff and equipment kept in readiness. All training sessions were supervised by an experienced SCI physiotherapist with expertise in $\mathrm{AD}$ and with detailed knowledge about the participant's triggers and initial symptoms during previous episodes of AD. In six of the eight training sessions, using a portable blood pressure monitor (CardioXplorer, Medical Information Technology Inc., Westwood, MA, USA) attached to the contralateral arm, blood pressure data and heart rate recordings were obtained with high temporal resolution (approx. every $5 \mathrm{~min}$ ) to monitor asymptomatic episodes of AD. In two training sessions where the original equipment was unavailable, another blood pressure monitor (Carescape V100, GE Healthcare, Chicago, IL, USA) with no recording function was applied manually approx. every $5 \mathrm{~min}$ for the same purpose. In addition, the supervising physiotherapist continuously asked the participant about symptoms related to AD. Immediately before and $\sim 30 \mathrm{~min}$ after both the first and last training sessions, blood samples were drawn from the left antecubital vein and subsequently analyzed for a range of biomarkers associated with DVT, rhabdomyolysis and vascular and nerve damage (for a listing of the analyzed biomarkers, see Table 2). Pain sensation (11-point Numeral Rating Scale (NRS)) was scored by the participant before and after cuff inflation and immediately after the last set of each exercise (i.e., preceding cuff release).

In addition, the participant underwent a 24-h-ABPM 2 months after the BFRE intervention period, using the same portable blood pressure monitor (recordings performed every $15-30 \mathrm{~min}$ ). From these 24 -h-ABPM recordings, data from (1) a conventional training session performed without BFR (note that the temporal resolution of the BP recordings in this free-flow training session was

Table 2 Blood profile of the SCI participant assessed immediately before and after the first and last training session, respectively.

\begin{tabular}{|c|c|c|c|c|c|c|c|}
\hline Analysis & Unit & Marker of & $\begin{array}{l}\text { Reference } \\
\text { interval }\end{array}$ & $\begin{array}{l}\text { Pre-first } \\
\text { training } \\
\text { session }\end{array}$ & $\begin{array}{l}\text { Post-first } \\
\text { training } \\
\text { session }\end{array}$ & $\begin{array}{l}\text { Pre-last } \\
\text { training } \\
\text { session }\end{array}$ & $\begin{array}{l}\text { Post-last } \\
\text { training } \\
\text { session }\end{array}$ \\
\hline Troponin $\mathrm{T}$ & $\mathrm{ng} / \mathrm{l}$ & $\begin{array}{l}\text { Acute coronary } \\
\text { syndromes }\end{array}$ & $<14$ & 113 & 114 & 107 & 103 \\
\hline Creatine-kinase & $\mathrm{U} / \mathrm{l}$ & $\begin{array}{l}\text { Muscle damage/ } \\
\text { rhabdomyolysis }\end{array}$ & $50-270$ & 319 & 318 & 288 & 286 \\
\hline Myoglobin & $\mu \mathrm{g} / \mathrm{l}$ & $\begin{array}{l}\text { Myoglubinuria/ } \\
\text { rhabdomyolysis }\end{array}$ & $<75$ & 80 & 71 & 41 & 45 \\
\hline Lactate-dehydrogenase & $\mathrm{U} / \mathrm{l}$ & Tissue damage & $105-205$ & 160 & 165 & 182 & 159 \\
\hline Protein & $\mathrm{g} / \mathrm{l}$ & & $62-78$ & 69 & 68 & 68 & 69 \\
\hline Potassium & $\mathrm{mmol} / \mathrm{l}$ & Hyperkalaemia & $3.5-4.6$ & 3.6 & 3.7 & 3.8 & 3.6 \\
\hline Sodium & $\mathrm{mmol} / \mathrm{l}$ & Hyponatremia & $137-145$ & 142 & 141 & 141 & 139 \\
\hline Free calcium-ions & $\mathrm{mmol} / \mathrm{l}$ & Cell damage/death & $1.18-1.32$ & 1.25 & 1.28 & 1.27 & 1.27 \\
\hline Albumin & $\mathrm{g} / 1$ & Hepatic stress & $34-45$ & 37 & 37 & 37 & 37 \\
\hline Creatinine & $\mu \mathrm{mol} / \mathrm{l}$ & Renal stress & $60-105$ & 43 & 40 & 41 & 46 \\
\hline $\begin{array}{l}\text { Estimated glomerular } \\
\text { Filtration rate (eGFR) }\end{array}$ & $\mathrm{ml} / \mathrm{min}$ & Renal stress & $>60$ & $>90$ & $>90$ & $>90$ & $>90$ \\
\hline C-reactive protein (CRP) & $\mathrm{mg} / \mathrm{l}$ & Inflammation & $<8$ & 31.9 & 30.1 & 5.7 & 5.8 \\
\hline Lymphocytes & $10^{9} / 1$ & $\begin{array}{l}\text { Immune system } \\
\text { dysfunction }\end{array}$ & $1.3-3.5$ & 1.57 & 1.42 & - & - \\
\hline Haemoglobin & $\mathrm{mmol} / \mathrm{l}$ & Anaemia & $8.1-10.3$ & 7.2 & 7.2 & 7.7 & 7.5 \\
\hline Thrombocytes & $10^{9} / 1$ & Vascular damage & $145-350$ & 234 & 238 & 291 & 287 \\
\hline Fibrin D-dimer & $\mathrm{mg} / \mathrm{l}$ & Thrombosis & $<0.5$ & 0.90 & $\mathrm{COA}$ & 0.87 & 1.0 \\
\hline
\end{tabular}

$\mathrm{COA}=$ Blood sample analysis unattainable due to coagulation of the sample. Reference intervals from a Danish database are based on healthy males aged $18-55$ years. 
lower than for the BFRE sessions) and (2) a sterile intermittent catheterization was extracted.

The study was reported to The Scientific Ethical Committees of Region Central Jutland (case no. 1-10-72-348-18), but the board deemed it unnecessary to obtain Ethical permission, as they considered the intervention regime as individual treatment initiative for a single patient. We certify that all applicable institutional and governmental regulations concerning the ethical use of human volunteers were followed during the course of this research. The participant gave verbal and written consent to participate in the study.

The training protocol was completed successfully and without any major modifications in the experimental procedures, with the exception of five intra-exercise cuff releases on spontaneous request of the participant. During eight training sessions and $341 \mathrm{~min}$ of supervised BFRE the participant experienced a single episode of symptomatic $\mathrm{AD}$, which he characterized as "beginning headache and discomfort" accompanied by a distinct autonomic response (SBP: +42 mmHg DBP: +46 mmHg HR: -21 bpm, compared to a recording performed $6 \mathrm{~min}$ prior to this event) following which the cuff was immediately released and the participant rested. The episode occurred during the last set of the last exercise in session 2 (Fig. 1); therefore, training was not resumed subsequently. Two minutes later, when the participant reported no remaining symptoms, a new blood pressure recording was performed and all values had normalized (Fig. 1). Subsequently, the participant resumed to
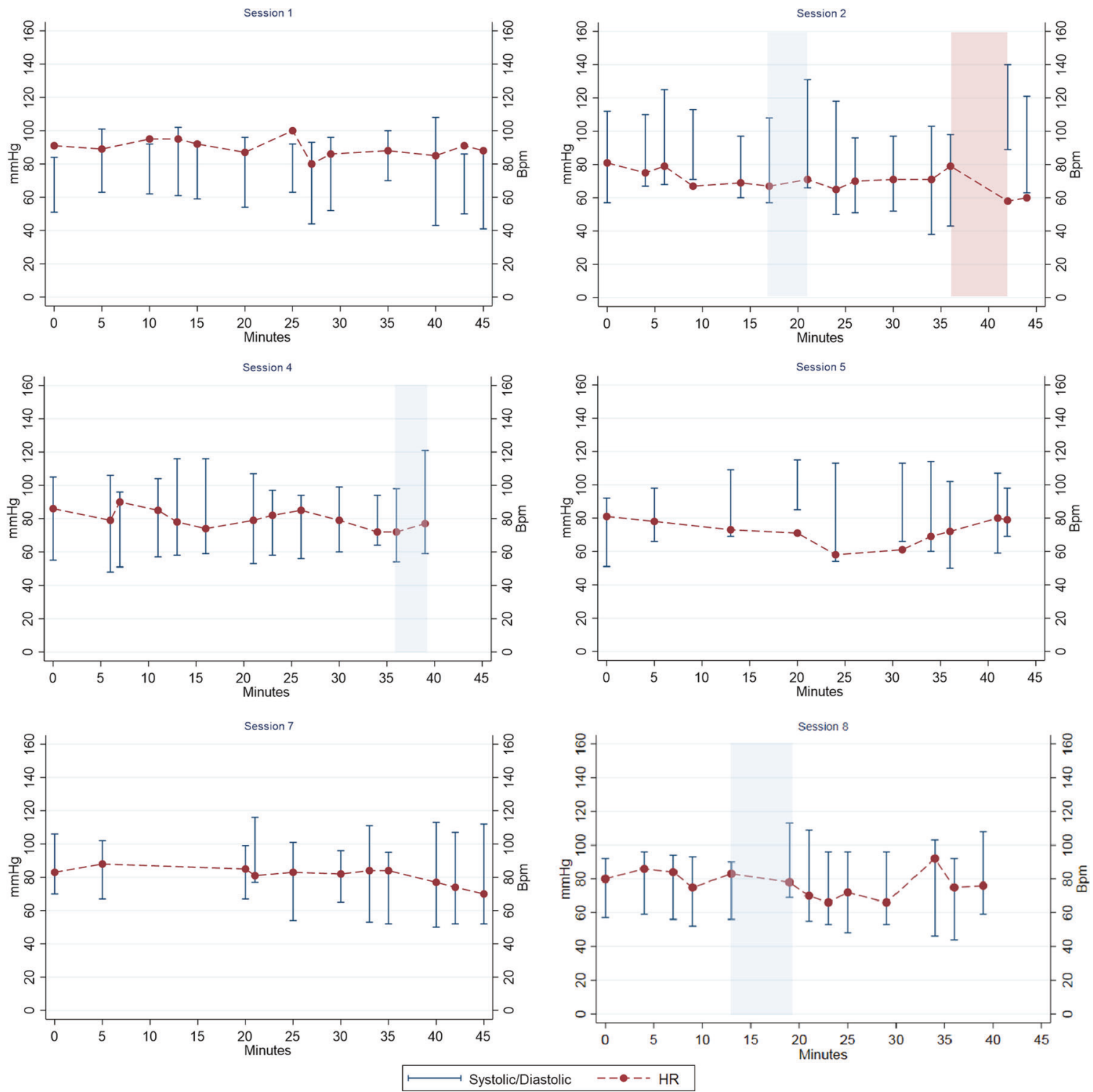

Fig. 1 Blood pressure and heart rate responses of the participant during six sessions of blood flow-restricted resistance training. SBP Systolic blood pressure, DBP Diastolic blood pressure, HR Heart

rate. Blue columns represent asymptomatic $\mathrm{AD}$ events, red column represents the symptomatic $\mathrm{AD}$ event. 
perform other clinical activities where he was kept under observation. Thus, the symptomatic episode that occurred during BFRE was reversed following $2 \mathrm{~min}$ of rest, similarly to as when $\mathrm{AD}$ episodes would trigger during conventional free-flow resistance training (Fig. 2). Here, the same physiotherapist would employ similar measurements and rest procedures with the participant.

A total of four cases of relatively rapid and short-lasting (4-6 min) SBP increases of 42, 23, 23 and $23 \mathrm{mmHg}$, respectively, were registered (of which one was the previously described symptomatic episode) during the duration of the study. These inherently classify as AD events according to the International Standards to Document Remaining Autonomic Function after Spinal Cord Injury (ISAFSCI) [19]. Developments of heart rate, systolic and diastolic blood pressure during training sessions with BFRE are presented in Fig. 1. Further, the reported AD events are marked in Figs. 1 and 2.

BFRE did not result in considerable changes in any biomarkers of DVT, rhabdomyolysis or vascular/nerve damage, as documented by the participant's blood profile (see Table 2).

The participant reported mild subjective pain during elbow extensors exercise, and moderate pain during elbow flexors exercise and wrist extensors exercise (see Table 3).

Two asymptomatic episodes of AD occurred in the single free-flow training session, based on ISAFSCI criterions [19] (Fig. 2).

24-h-ABPM recordings from during catheterization also revealed a severe episode of $\mathrm{AD}$ that was likely induced by bladder distension (SBP: $+101 \mathrm{mmHg}$; DBP: $+85 \mathrm{mmHg}$;

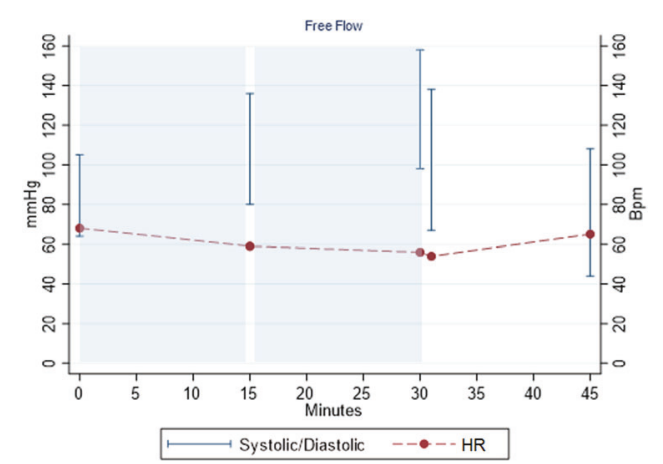

Fig. 2 Blood pressure and heart rate responses of the participant during a free-flow training session. SBP Systolic blood pressure, DBP Diastolic blood pressure, HR Heart rate. Blue columns represent asymptomatic $\mathrm{AD}$ events.
HR: $+175 \mathrm{bpm}$ (from $58 \rightarrow 233 \mathrm{bpm}$ ), compared to the preceding record $30 \mathrm{~min}$ prior to catheterization).

\section{Discussion}

In the present study, a 23-year-old tetraplegic male with autonomic dysfunction including dysreflexia successfully completed 4 weeks of BFR resistance exercise for the upper extremities. However, several episodes of $\mathrm{AD}$ were observed. Across eight training sessions, a single episode of symptomatic $\mathrm{AD}$ was registered, while three additional episodes of asymptomatic $\mathrm{AD}$ were registered according to the ISAFSCI definitions [19]. Correspondingly, two asymptomatic $\mathrm{AD}$ episodes were recorded during a single control (i.e., free-flow) training session.

BFRE did not result in any marked, acute changes in the participant's blood profile, neither following the first or the last training sessions, respectively (Table 2). Collectively evaluated, the blood sample data seemed affected by the participant's general state of health, and was potentially influenced from hip surgery procedures performed 8 weeks prior to initiation of the training study. Furthermore, we were unable to perform D-dimer analysis on blood samples obtained following the first training session, due to partial coagulation of the samples during transport to the laboratory. It may be argued, therefore, that the blood sample results are somewhat inconclusive. The participant reported mean pain levels corresponding to mild, moderate and mild pain in the classifications of the NRS [20], during the first (triceps extensions), second (biceps curls) and third (wrist extensions) exercise, respectively (Table 3). As the participant presented with impaired elbow extensor function (MMT 2+/2), normal elbow flexor function (MMT 5/5) and moderate wrist extensor function (MMT 3/3), it could be suggested that the level of reported pain is associated with the amount of motor function remaining (and hence magnitude of force production) in the respective muscle groups. In a recent study by Martin-Hernandez et al. [21], ablebodied subjects reported "strong" to "very strong" pain during six BFRE sessions of the lower limbs, while less pain has been noted when subjects gradually become accustomed to the BFRE training protocol [22]. Although the results are not readily comparable, as different rating scales and different muscle groups were employed between studies, it is interesting to note that perceptual pain during

Table 3 Mean pain scores of the SCI participant (11-point numerical rating scale) obtained before and after cuff inflation and immediately after the last set of each exercise (before deflation).

\begin{tabular}{llllll}
\hline & Before inflation & After inflation & $\begin{array}{l}\text { After first exercise } \\
\text { Triceps ext. }\end{array}$ & $\begin{array}{l}\text { After second exercise } \\
\text { Biceps Curls }\end{array}$ & $\begin{array}{l}\text { After third exercise } \\
\text { Wrist ext. }\end{array}$ \\
\hline Reported pain & 0.1 & 1.1 & 1.4 & 6.4 & 4.9 \\
\hline
\end{tabular}


BFRE was reported to be higher in able-bodied individuals as compared to the present participant. However, whether this is due to lower exercise intensity (load/training volume) and/or muscle volume activation, or due to SCI-induced hypoalgesia in the present participant remains unknown, and warrants further attention. A variety of noxious or nonnoxious stimuli below the site of the spinal lesion can elicit $\mathrm{AD}$ [15]. In the present case, BFRE-induced AD is possible due to the participant not being able to register nociceptive signals from the trained limb. Both Martin-Hernandez et al. [21] and Nielsen et al. [22] reported that the mean reported pain level was lower for the last training session as compared to the first. A similar adaptive effect was not seen during the BFRE intervention period in the present case study, where the level of discomfort remained relatively stable throughout the 4-week training period. It should be noted, however, that in spite of the relatively low perceptual pain responses reported by our participant, on five occasions the cuff had to be released (deflated) following the second set of the second exercise (biceps curls, during which the reported pain was highest). The participant reported "a burning sensation in his biceps muscle". The symptoms ceased immediately following cuff deflation and rest, as also observed in able-bodied individuals [22]. Thus, it appears that the reported discomfort was naturally occurring muscle pain from the exercise performed. When asked if wishing to abort the training session (this was asked following each spontaneous deflation) the participant declined. The deflation requests did not seem to correspond with the timing of the AD events.

In conclusion, the present study participant (23-yr-old tetraplegic male with autonomic dysfunction including dysreflexia) was able to perform and tolerate 4 weeks of BFRE, although encountering four episodes of AD during the exercise sessions, of which one was symptomatic. In addition, two AD episodes were observed during a single control (conventional free-flow) resistance training session. The participant reported mild to moderate pain during the BFRE training sessions, and the discomfort ceased immediately following cuff release. Whether other types of BFRE protocols can be applied without inducing AD in SCI patients with a prior history of $\mathrm{AD}$ remains unknown. Future studies on BFRE in SCI individuals with injury at or above T6 should address the safety of BFRE training in this patient population, particularly in individuals with indication of $\mathrm{AD}$.

Acknowledgements The authors would like to thank the participant for his effort and patience during the project.

Author contributions $\mathrm{SK}, \mathrm{ABJ}$ and $\mathrm{HK}$ are responsible for concept, design and management of the study. SK performed data analyses and wrote the initial draft of the paper. JV supervised training and collected data. KES, PA, and HK provided guidance and revised the paper. All authors have read and accepted the final version of the paper.

\section{Compliance with ethical standards}

Conflict of interest The authors declare that they have no conflict of interest.

Publisher's note Springer Nature remains neutral with regard to jurisdictional claims in published maps and institutional affiliations.

\section{References}

1. Biering-Sørensen F, Bickenbach JE, El Masry WS, Officer A, von Groote PM. ISCoS-WHO collaboration. International Perspectives of Spinal Cord Injury (IPSCI) report. Spinal Cord. 2011;49:679-83.

2. Anderson KD. Targeting recovery: priorities of the spinal cordinjured population. J Neurotrauma. 2004;21:1371-83.

3. Snoek GJ, Ijzerman MJ, Hermens HJ, Maxwell D, BieringSorensen F. Survey of the needs of patients with spinal cord injury: impact and priority for improvement in hand function in tetraplegics. Spinal Cord. 2004;42:526-32.

4. Simpson LA, Eng JJ, Hsieh JTC, Wolfe DL, Program GC. The health and life priorities of individuals with spinal cord injury: a systematic review. J Neurotrauma. 2013;29:1548-55.

5. Spooren AIF, Janssen-Potten YJM, Snoek GJ, Ijzerman MJ, Kerckhofs E, Seelen HAM. Rehabilitation outcome of upper extremity skilled performance in persons with cervical spinal cord injuries. J Rehabilit Med. 2008;40:637-44.

6. Slysz J, Stultz J, Burr JF. The efficacy of blood flow restricted exercise: a systematic review \& meta-analysis. J Sci Med Sport. 2016;19:669-75.

7. Jørgensen AN, Aagaard P, Nielsen JL, Frandsen U, Diederichsen LP. Effects of blood-flow-restricted resistance training on muscle function in a 74-year-old male with sporadic inclusion body myositis: a case report. Clin Physiol Funct Imaging. 2016;36:504-9.

8. Gualano B, Neves M, Lima FR, Pinto ALDS, Laurentino G, Borges $\mathrm{C}$, et al. Resistance training with vascular occlusion in inclusion body myositis: a case study. Med Sci Sports Exerc. 2010;42:250-4.

9. Salvador AF, Schubert KR, Cruz RS de O, Corvino RB, Pereira $\mathrm{KL}$, Caputo F, et al. Bilateral muscle strength symmetry and performance are improved following walk training with restricted blood flow in an elite paralympic sprint runner: case study. Phys Ther Sport. 2016;20:1-6.

10. Grutter Lopes K, Alexandre Bottino D, Farinatti P, Coelho De Souza M das G, Alves Maranhão P, De Araujo CMS, et al. Strength training with blood flow restriction-a novel therapeutic approach for older adults with sarcopenia? A case report. Clin Interv Aging. 2019;14:1461-9.

11. Gorgey AS, Timmons MK, Dolbow DR, Bengel J, Fugate-Laus $\mathrm{KC}$, Michener LA, et al. Electrical stimulation and blood flow restriction increase wrist extensor cross-sectional area and flow meditated dilatation following spinal cord injury. Eur J Appl Physiol. 2016;116:1231-44.

12. Wernbom M, Paulsen G, Bjørnsen T, Cumming K, Raastad T. Risk of Muscle Damage With Blood Flow-Restricted Exercise Should Not Be Overlooked. Clin J Sport Med. 2019;00:1.

13. Nakajima T, Kurano M, Iida H, Takano H, Oonuma H, Morita T, et al. Use and safety of KAATSU training:Results of a national survey. Int J KAATSU Train Res. 2006;2:5-13. 
14. Clark BC, Manini TM, Hoffman RL, Williams PS, Guiler MK, Knutson MJ, et al. Relative safety of 4 weeks of blood flowrestricted resistance exercise in young, healthy adults. Scand J Med Sci Sport. 2011;21:653-62.

15. Krassioukov A, Warburton DE, Teasell R, Eng JJ. A systematic review of the management of autonomic dysreflexia after spinal cord injury. Arch Phys Med Rehabilit. 2009;90:682-95.

16. Stavres J, Singer TJ, Brochetti A, Kilbane MJ, Brose SW, Mcdaniel J. The feasibility of blood flow restriction exercise in patients with incomplete spinal cord. Injury. 2018;10:1368-79.

17. Hubli M, Gee CM, Krassioukov AV. Refined assessment of blood pressure instability after spinal cord injury. Am J Hypertens. 2015;28:173-81.

18. Takarada Y, Takazawa H, Sato Y, Takebayashi S, Tanaka Y, Ishii $\mathrm{N}$. et al. Effects of resistance exercise combined with moderate vascular occlusion on muscular function in humans. J Appl Physiol (1985). 2000;88:2097-106.
19. Alexander MS, Biering-Sorensen F, Bodner D, Brackett NL, Cardenas D, Charlifue S, et al. International standards to document remaining autonomic function after spinal cord injury. Spinal Cord. 2008;47:36.

20. Boonstra AM, Stewart RE, Albère AJ, René RF, Swaan JL, Schreurs KMG, et al. Cut-offpoints for mild, moderate, and severe pain on the numeric rating scale for pain in patients with chronic musculoskeletal pain: variability and influence of sex and catastrophizing. Front Psychol. 2016;7:1-9.

21. Martín-Hernández J, Ruiz-Aguado J, Herrero AJ, Loenneke JP, Aagaard P, Cristi-Montero C, et al. Adaptation of perceptual responses to low-load blood flow restriction training. J Strength Cond Res. 2017;31:765-72.

22. Nielsen JL, Frandsen U, Prokhorova T, Bech RD, Nygaard T, Suetta C, et al. Delayed effect of blood flow-restricted resistance training on rapid force capacity. Med Sci Sports Exerc. 2017;49:1157-67. 